\title{
Probable trastorno de ansiedad generalizada en la pandemia COVID-19: valoración en médicos generales del Caribe colombiano
}

\section{Probable generalized anxiety disorders in the COVID-19 pandemic: assessment in general practitioners in the Colombian Caribbean}

\author{
Álvaro Monterrosa-Castro (D) 1 , Camila Buelvas-de-la-Rosa (iD)2 ${ }^{2}$ Estefana Ordosgoitia-Parra (iD) ${ }^{3}$
}

1. Universidad de Cartagena. Cartagena, Colombia. Correo: alvaromonterrosa@gmail.com - https://orcid.org/0000-0002-0686-6468

2. Universidad de Cartagena. Cartagena, Colombia. Correo: camilabuelvas@ hotmail.com - https://orcid.org/0000-0001-8675-250X

3. Universidad de Cartagena. Cartagena, Colombia. Correo: estefanaop@hotmail.com - https://orcid.org/0000-0002-4264-7836

Tipología: Artículo de investigación científica y tecnológica

Para citar este artículo: Monterrosa-Castro A, Buelvas-de-la-Rosa C, Ordosgoitia-Parra E. Probable trastorno de ansiedad generalizada en la pandemia COVID-19: valoración en médicos generales del Caribe colombiano. Duazary. 2021 enero; 18(1):7-19. Doi: https://doi.org/10.21676/2389783X.3890

Recibido en mayo 15 de 2020

Aceptado en noviembre 19 de 2020

Publicado en línea en enero 07 de 2021

\section{RESUMEN}

Palabras

clave:

trastornos de

ansiedad;

ansiedad de

desempeño;

miedo; estrés

psicológico;

infecciones

por

médicos

generales. coronavirus;

La pandemia del COVID-19 es la más reciente en la historia de la humanidad, caracterizada por elevadas implicaciones en términos de mortalidad, morbilidad física y psicológica. El objetivo de esta investigación fue identificar pensamientos, percepciones, sentimientos, subjetividades y actuaciones con referencia al desempeño médico y la pandemia del COVID-19, así como estimar su asociación con probables trastornos de ansiedad generalizada [TAG]. Se realizó un estudio transversal en médicos generales del Caribe colombiano, por medio de invitación y participación electrónica para diligenciar un formulario que contenía características sociodemográficas y 41 interrogantes sobre pensamientos, sentimientos, percepciones, subjetividades o actuaciones relacionadas con el COVID-19, tomadas de una lluvia de ideas sobre pandemias y salud mental. Se aplicó Generalized Anxiety Disorder 7-item Scale [GAD-7] para identificar probable TAG. Se realizó regresión logística no ajustada. Participaron 294 médicos generales, 59,6\% femeninas, el 83\% laboraba en capitales de departamentos. El 38,4\% presentó TAG y más de la mitad manifestó temor al COVID-19. A la presencia de TAG se asociaron los síntomas: estrés, nerviosismo, temor, cansancio, síntomas virales, incomodidad con respecto a la pandemia, decepción laboral y percepción de discriminación. A menor presencia de TAG se asociaron: sentirse protegido por los empleadores, percibir suficientes las medidas gubernamentales y creer en la información de las autoridades.

\section{ABSTRACT}

Keywords:

Anxiety

Disorders;

Performance

Anxiety;

Fear;

Psychologica

I Stress;

Coronavirus

Infections;

General

Practitioners
The COVID-19 pandemic is the most recent in history, characterized by high implications in terms of mortality, physical and psychological morbidity. This research aimed to identify thoughts, perceptions, feelings, subjectivities, and actions concerning medical performance and the COVID-19 pandemic, as well as to estimate its association with probable Generalized Anxiety Disorders [GAD]. A cross-sectional study was carried out among general practitioners in the Colombian Caribbean, using electronic invitation and participation to fill out a form containing sociodemographic characteristics and 41 questions about thoughts, feelings, perceptions, subjectivities, or actions related to COVID-19, taken from brainstorming on pandemics and mental health. The Generalized Anxiety Disorder 7-item Scale [GAD-7] was applied to identify probable GAD. Unadjusted logistic regression was performed. A total of 294 general practitioners participated, $59.6 \%$ female, $83 \%$ of whom worked in departmental capitals. $38.4 \%$ had probable GAD and more than half expressed fear of COVID-19. Symptoms associated with the presence of GAD included stress, nervousness, fear, fatigue, viral symptoms, discomfort with the pandemic, job 
disappointment, and perception of discrimination. A lower presence of GAD was associated with the feeling of protection by their employers, perception of sufficient government measures, and trust in the information of the authorities.

\section{INTRODUCCIÓN}

A finales de diciembre de 2019 se observaron casos de neumonía atípica de etiología desconocida en Wuhan, China. El cuadro clínico estaba compuesto por fiebre, tos no productiva, mialgias, diarrea o anosmia ocasional, fatiga, disnea progresiva, frecuente leucopenia y alteración del patrón imagenológico pulmonar, y fue denominado Coronavirus Disease 2019 o COVID-19, que corresponde al acrónimo en lengua inglesa. Un mes más tarde se identificó al SARS-CoV-2 como el patógeno causante ${ }^{1,2}$.

El brote infeccioso se propagó rápidamente hasta alcanzar niveles de pandemia. En consecuencia, el 11 de marzo del 2020, la Organización Mundial de la Salud $^{3-6}$ declaró la emergencia de salud pública de interés internacional [PHEI, por sus siglas en inglés]. En lo sucesivo, las autoridades académicas y los organismos asesores en salud señalaron acciones para afrontar los retos sanitarios, dada la elevada morbilidad y mortalidad ${ }^{7,8}$. Las recomendaciones iniciales fueron: lavado exhaustivo de manos, uso de tapabocas, distanciamiento social y confinamiento preventivo $^{4,9,10}$.

Algunos autores ${ }^{11-15}$ han documentado afectación emocional, estigmatización y temor de las personas y los profesionales de la salud en momentos de brotes epidémicos o pandemias. Médicos y enfermeras de hospitales chinos que atendieron a pacientes con COVID-19 informaron altas tasas de síntomas depresivos, ansiedad, insomnio y angustia ${ }^{16}$.

El miedo es una emoción adaptativa: ayuda a la defensa ante un peligro potencial. La sobrestimación en magnitud y tiempo es una carga para la vida diaria y puede conllevar a enfermedad postraumática, trastorno de estrés y otros trastornos de ansiedad ${ }^{17,18}$. El Trastorno de Ansiedad Generalizada [TAG] se caracteriza por preocupación persistente e incontrolable que se producen consistentemente; la evolución es fluctuante y empeora durante el estrés. Se ha indicado que la mayoría de los pacientes pueden presentar uno o más de otros trastornos psiquiátricos asociados a depresión mayor, fobia específica, fobia social, trastorno de angustia y de la salud en general o dolor corporal inespecífico, que interfieren en sus rutinas diarias. Este trastornó muy pocas veces es diagnosticado tempranamente por lo que tiene un alto número de solicitudes a visitas médicas ${ }^{17-20 .}$ La herramienta Generalized Anxiety Disorder 7-item Scale [GAD-7] es ampliamente aceptada para realizar cribado e identificar casos de probable $T_{A G}{ }^{21,22}$. Hacen falta estudios que valoren la ansiedad y los aspectos psicosociales en profesionales colombianos del área de la salud bajo condiciones de pandemia. El objetivo fue identificar la frecuencia de respuesta de varios interrogantes que exploran pensamientos, percepciones, sentimientos, subjetividades y actuaciones en referencia al desempeño médico y la pandemia del COVID-19, así como estimar su asociación con TAG en médicos generales del Caribe colombiano.

\section{MATERIALES Y MÉTODOS}

\section{Tipo de investigación}

Estudio transversal que hace parte del proyecto DISEU [Dinámica Psicosocial en Universitarios, brazo COVID-19]. El presente es un análisis parcial de los datos aportados electrónicamente por un grupo de médicos generales que ejercen en Colombia [datos no disponibles aún] y considera la información entregada por los que manifestaron laborar en el Caribe colombiano, estando el país bajo cuarentena decretada por el gobierno nacional.

\section{Participantes}

Se convocaron por redes sociales [Instagram, Facebook y WhatsApp] y correos electrónicos personales o de agremiaciones a médicos generales para que diligenciaran entre el primero y cinco de abril del 2020 un formulario especialmente 
diseñado. Se consideraron como criterios de inclusión: ejercer labor médica asistencial en marzo del mismo año, laborar en ambientes ambulatorios u hospitalarios, realizar cuidado sanitario en cualquiera de los niveles de complejidad de atención, tener vinculación con instituciones públicas o privadas $\mathrm{y}$ atender pacientes con cualquier tipo de afectación o condición general de salud. Se determinó como único criterio de exclusión que los participantes no diligenciaran completamente el formulario.

\section{Instrumentos}

Se diseñó el formulario electrónico con Google Forms ( $\mathbb{C}$, de uso frecuente en ámbitos educativos y sociales, de fácil diligenciamiento, de uso libre y con las ventajas de la virtualidad, para recabar comentarios y generar datos estadísticos. Se solicitó a los participantes aplicar sus respuestas al lapso 2430 de marzo del 2020.

El formulario estaba conformado por un grupo de preguntas. Las primeras interrogaban características sociodemográficas [género, edad y municipio donde laboraban]. Luego había un listado de interrogantes escogidos de una lluvia de ideas de conceptos teóricos de la ansiedad, basados en textos y artículos $^{17-20}$. Las preguntas interrogaban sobre pensamientos, percepciones, sentimientos, subjetividades y actuaciones en referencia a su desempeño médico y la pandemia del COVID-19, para contestar Sí/No. Finalmente, se aplicaron las preguntas de Generalized Anxiety Disorder 7-item Scale [GAD-7], la cual es una herramienta de cribado, utilizada para evaluar probable TAG en contextos clínicos y poblacionales. Cada pregunta se responde tipo Likert: cero [Nunca], uno [Menos de la mitad de los días], dos [Más de la mitad de los días] y tres [Casi todos los días]. Se obtiene puntuación total entre $0-21$; con 0-4 puntos no se aprecia ansiedad, 5-9 indica ansiedad leve, 10-14 moderada y 15-21 severa. Puntuación de diez o superior define presencia de probable TAG. GAD-7 ha sido validada en diferentes poblaciones $y$ lenguas, mostrando adecuada consistencia y fiabilidad $^{21,22}$.

\section{Procedimiento}

Al parecer los datos del número de médicos que ejercen medicina general en Colombia son contradictorios. Un informe de prensa ${ }^{23}$ señaló en el 2011 que se contaban 77.473 médicos generales; otro $^{24}$ en el 2018 informó de 65 939. Datos del Observatorio Laboral para la Educación Nacional indicó a un medio periodístico ${ }^{25}$ que entre 20012018 se habían graduado 73092 médicos, 26\% especialistas, y se estimaron 54092 los médicos generales egresados en ese período. Tampoco es clara la proporción de los que laboran en la región Caribe colombiana. Con la calculadora Netquest se estimaron 385 participantes, heterogeneidad 50\%, nivel de confianza $95 \%$ y margen de error $5 \%$. Se incluirán en el estudio todos los que informen laborar en la región Caribe del país.

Desde la plataforma de Google @ se descargó la base de datos que se genera automáticamente y se realizó el análisis con EPI-INFO-7 [CDC, Atlanta, EE. UU.]. Los datos continuos se expresan en media y desviación estándar; los categóricos en absolutos, porcentajes e IC95\%. Se adelantó regresión logística no ajustada entre las características sociodemográficas, así como pensamientos, sentimientos, percepciones, subjetividades y actuaciones referentes al desempeño médico y al COVID-19 [variables independientes] con TAG [variable dependiente], $p<0,05$ : estadísticamente significativo.

\section{Declaración sobre aspectos éticos}

Para conservar el anonimato, al descargar la base de datos desde la plataforma se eliminó el email de los participantes. Los remitentes al diligenciarlo aceptaban términos y concedían consentimiento informado, según exigencias de la declaración de Helsinki, 1975. Se tuvieron en cuenta normas técnico/científicas para investigación en salud, Resolución 8430-1993, que otorga al presente diseño clasificación de riesgo mínimo ${ }^{26}$. El proyecto DISEU y el Grupo están avalados por Universidad de Cartagena, Colombia, Acta 064-2019, Resolución 01430-2019 del plan de fortalecimiento y sostenibilidad a grupos de investigación. 


\section{RESULTADOS}

Se recibieron vía electrónica 531 formularios adecuadamente diligenciados, 37,9\% por encima del tamaño de la muestra. De estos, 294 (55,3\%) informaron laborar en el Caribe y con ellos se realizó el estudio. Edad media 33,0 $\pm 9,3$ años, la mitad entre 21-30 años y seis de cada diez eran médicas. 244 $(83,0 \%)$ laboraban en capital departamental (Tabla 1).

Tabla 1. Características sociodemográficas.
Las respuestas a las preguntas para explorar pensamientos, percepciones, sentimientos, subjetividades y actuaciones en referencia al desempeño médico y al COVID-19 se encuentran en la Tabla 2. Cuatro de cada diez creyeron tener síntomas del COVID-19. Más del 68\% temían ser portadores asintomáticos o perder la vida, mientras que más del $80 \%$ temían acudir como pacientes a un centro sanitario o llevar el virus a casa. Un mayor número de médicos informaron jaquecas, cansancio extremo, incomodidad o estaban nerviosos al ver noticias $(p<0,05)$.

\begin{tabular}{|l|l|c|}
\hline \multirow{4}{*}{ Género } & Variables & $\mathbf{n}(\%)[$ IC95 \%] \\
\hline \multirow{5}{*}{ Rango etario } & Masculino & $119(40,4)[34,8-46,3]$ \\
\cline { 2 - 3 } & Femenino & $175(59,6)[53,6-65,1]$ \\
\cline { 2 - 3 } & $21-30$ & $146(49,6)[43,8-55,5]$ \\
\cline { 2 - 3 } & $31-40$ & $97(32,9)[27,6-38,6]$ \\
\cline { 2 - 3 } & $41-50$ & $26(8,9)[5,8-12,6]$ \\
\cline { 2 - 3 } & $51-60$ & $18(6,3)[3,6-9,5]$ \\
\cline { 2 - 3 } & $61-70$ & $7(2,3)[0,9-4,8]$ \\
\hline \multirow{5}{*}{ Ubicación Geográfica } & Ciudad no capital & $50(17,0)[12,8-21,8]$ \\
\cline { 2 - 3 } & Ciudad capital & $244(83,0)[78,2-87,1]$ \\
\hline & Atlántico & $32(10,8)[7,5-15,0]$ \\
\cline { 2 - 3 } & Bolívar & $201(68,4)[62,7-73,6]$ \\
\cline { 2 - 3 } & Cesar & $9(3,1)[1,4-5,7]$ \\
\cline { 2 - 3 } & Córdoba & $16(5,4)[3,1-8,6]$ \\
\cline { 2 - 3 } & Guajira & $3(1,0)[0,2-2,9]$ \\
\cline { 2 - 3 } & Magdalena & $19(6,5)[3,9-9,9]$ \\
\cline { 2 - 3 } & Sucre & $14(4,8)[2,6-7,8]$ \\
\hline & Barranquilla & $29(11,9)[7,7-16,1]$ \\
\cline { 2 - 3 } & Cartagena & $174(71,4)[65,2-76,9]$ \\
\cline { 2 - 3 } & Montería & $9(3,6)[1,7-6,8]$ \\
\cline { 2 - 3 } & Santa Marta & $18(7,4)[4,4-11,4]$ \\
\cline { 2 - 3 } & Valledupar & $7(2,9)[4,2-4,9]$ \\
\cline { 2 - 3 } & Riohacha & $1(0,4)[0,0-2,2]$ \\
\cline { 2 - 3 } & Sincelejo & $6(2,4)[0,9-5,2]$ \\
\hline & &
\end{tabular}

El alfa de Cronbach de GAD-7: 0,793 y la puntuación $8,30 \pm 5,41$. No presentaron ansiedad: 78 (26,5\%) [IC95\%:21,5-31,9]. Ansiedad leve: 103 (35,0 \%) [IC95\%:29,5-40,7], moderada: 73 (24,8\%) [IC95\%:20,0-30,1] y severa: 40 (13,6\%) [IC95 \%:9,918,0]. Se encontró probable TAG en $113(38,4 \%)$ [IC95\%:32,8-44,2] y no lo presentaron 181 (61,5\%) [IC95\%:55,7-67,7] $(p<0,05)$ (Tabla 3).
En la regresión logística no ajustada se observó que ser médica se asoció con tres veces probable TAG. Sentirse decepcionado con el trabajo o percibir discriminación se asoció con dos veces probable TAG, mientras que sentirse protegido por el empleador se asoció con reducción del 53\% (Tabla 4). Laborar en cualquier ciudad, temer contagiarse 
en el trabajo o convivir con familiares en alto riesgo no se asociaron con probable TAG $(p>0,05)$.

Tabla 2. Exploración de pensamientos, percepciones, sentimientos, subjetividades y actuaciones en referencia al COVID-19.

\begin{tabular}{|c|c|c|}
\hline \multirow{2}{*}{ Interrogantes } & Si & No \\
\hline & \multicolumn{2}{|c|}{$\mathrm{n}(\%)$ [IC95\%] } \\
\hline Haber creído tener síntomas de COVID-19 & $132(44,9)[39,1-50,7]$ & $162(55,1)[49,2-60,8]$ \\
\hline El número de casos informados por las autoridades era confiable & $20(6,8)[4,20-10,3]$ & $274(93,2)[89,6-95,8]$ \\
\hline El número de pruebas realizadas eran suficientes & $15(5,1)[2,88-8,28]$ & $279(94,9)[91,7-97,1]$ \\
\hline Temor de llegar a la mortalidad de China & $270(91,8)[88,1-94,7]$ & $24(8,2)[5,30-11,9]$ \\
\hline Las medidas gubernamentales adoptadas eran suficientes & $62(21,1)[16,5-26,2]$ & $232(78,9)[73,8-83,4]$ \\
\hline Su comunidad acataba el confinamiento obligatorio & $6(2,1)[0,75-4,39]$ & $288(97,9)[95,6-99,2]$ \\
\hline El equipo de salud del país era suficiente & $31(10,6)[7,2-14,6]$ & $263(89,4)[85,3-92,7]$ \\
\hline Temían la necesidad de acudir como paciente & $247(84,1)[79,3-88,0]$ & $47(15,9)[11,9-20,6]$ \\
\hline Satisfacción con su labor profesional médica & $221(75,1)[69,8-80,0]$ & $73(24,9)[20,0-30,1]$ \\
\hline Sentirse protegido por su empleador & $52(17,7)[13,5-22,5]$ & $242(82,3)[77,4-86,5]$ \\
\hline Contribuir a mejorar la situación de pandemia & $265(90,1)[86,1-93,2]$ & $29(9,9)[6,71-13,8]$ \\
\hline Angustiado al pensar que debía trabajar al día siguiente & $212(72,1)[66,6-77,1]$ & $82(27,9)[22,8-33,3]$ \\
\hline Temía contagiarse con el virus en el trabajo & $286(97,2)[94,7-98,8]$ & $8(2,8)[1,1-5,2]$ \\
\hline Pensó renunciar al trabajo para proteger la familia & $150(51,0)[45,1-56,8]$ & $144(49,0)[43,1-54,8]$ \\
\hline Decepcionado en su trabajo como médico & $182(61,9)[56,0-67,4]$ & $112(38,1)[32,5-43,9]$ \\
\hline Percepción de discriminación por su condición de médico & $132(44,9)[39,1-50,7]$ & $162(55,1)[49,2-60,8]$ \\
\hline Protocolo exhaustivo de desinfección al llegar a casa & $272(92,5)[88,8-95,2]$ & $22(7,5)[4,7-11,1]$ \\
\hline Temor de llevar el virus a su casa & $283(96,2)[93,4-98,1]$ & $11(3,8)[1,8-6,6]$ \\
\hline Temor en la familia que regrese a casa infectado del COVID-19 & $241(81,9)[77,0-86,2]$ & $53(18,1)[13,8-22,9]$ \\
\hline Convive con familiares de alto riesgo para enfermedad severa & $190(64,6)[58,8-70,0]$ & $104(35,4)[29,9-41,1]$ \\
\hline Siente temor de ser portador asintomático del COVID-19 & $202(68,7)[63,0-73,9]$ & $92(31,3)[26,0-36,9]$ \\
\hline Contemplar cambiar de casa para proteger la familia & $212(72,1)[66,6-77,1]$ & $82(27,9)[22,8-33,3]$ \\
\hline Pesadillas con el COVID-19 & $194(65,9)[60,2-71,3]$ & $100(34,1)[28,6-39,7]$ \\
\hline Estresado desde que se inició la pandemia & $230(78,2)[73,0-82,8]$ & $64(21,8)[17,1-26,9]$ \\
\hline Intranquilidad desde que se inició la pandemia & $70(23,9)[19,0-29,1]$ & $224(76,1)[70,9-80,9]$ \\
\hline Dificultad para dormir & $181(61,5)[55,7-67,1]$ & $113(38,5)[32,8-44,2]$ \\
\hline Jaquecas, dolor de cabeza & $172(58,5)[52,6-64,1]$ & $122(41,5)[35,8-47,3]$ \\
\hline Indigestión, molestias gastrointestinales & $155(53,0)[47,1-58,9]$ & $137(47,0)[41,0-52,8]$ \\
\hline Extremadamente cansado, agotado & $181(61,5)[55,7-67,1]$ & $113(38,5)[32,8-44,2]$ \\
\hline Tendencia a comer, beber o fumar, más de lo habitual & $174(59,1)[53,3-64,8]$ & $120(40,9)[35,1-46,6]$ \\
\hline Disminución del interés sexual & $156(53,0)[47,1-58,8]$ & $138(47,0)[41,1-52,8]$ \\
\hline Respiración entrecortada o ahogo & $210(71,5)[65,9-76,5]$ & $84(28,5)[23,4-34,1]$ \\
\hline Disminución del apetito & $218(74,1)[68,7-79,0]$ & $76(25,9)[20,9-31,2]$ \\
\hline Temblores musculares o tics nerviosos & $241(81,9)[77,0-86,2]$ & $53(18,1)[13,8-22,9]$ \\
\hline Pinchazos dolorosos en el cuerpo & $202(68,7)[63,0-73,9]$ & $92(31,3)[26,0-36,9]$ \\
\hline No desear levantarse en la mañana para ir a trabajar & $154(52,3)[46,5-58,2]$ & $140(47,7)[41,7-53,5]$ \\
\hline Mayor humedad en manos & $83(28,3)[23,1-33,7]$ & $211(71,7)[46,5-58,2]$ \\
\hline Mucho temor al COVID-19 & $247(84,0)[79,3-88,0]$ & $47(16,0)[11,9-20,6]$ \\
\hline Incomodidad al pensar en el COVID-19 & $221(75,1)[69,8-80,0]$ & $73(24,9)[20,0-30,1]$ \\
\hline Temor de perder la vida por el COVID-19 & $209(71,0)[65,5-76,2]$ & $85(29,0)[23,8-34,4]$ \\
\hline Nerviosismo con las noticias sobre el COVID-19 & $200(68,0)[62,3-73,3]$ & $94(32,0)[26,6-37,6]$ \\
\hline
\end{tabular}




\begin{tabular}{|l|c|c|c|c|c|}
\hline \multicolumn{1}{|c|}{ Ítems } & \multicolumn{1}{|c|}{ Nunca } & $\begin{array}{c}\text { Menos de la mitad de } \\
\text { los días }\end{array}$ & $\begin{array}{c}\text { Más de la mitad } \\
\text { de los días }\end{array}$ & $\begin{array}{c}\text { Casi todos los } \\
\text { días }\end{array}$ \\
\cline { 2 - 5 } & \multicolumn{4}{|c|}{$\mathbf{n ( \% ) [ I C 9 5 \% ]}$} \\
\hline $\begin{array}{l}\text { Sentirse nervioso o } \\
\text { alterado }\end{array}$ & $94(31,9)[26,6-37,6]$ & $124(42,1)[36,4-48,0]$ & $49(16,6)[12,5-21,4]$ & $\begin{array}{c}27(9,1)[6,14- \\
13,0]\end{array}$ \\
\hline $\begin{array}{l}\text { No poder dejar de } \\
\text { preocuparse }\end{array}$ & $73(24,8)[20,0-30,1]$ & $118(40,1)[34,4-45,9]$ & $56(19,0)[14,7-24,0]$ & $\begin{array}{c}47(15,9)[11,9- \\
20,6]\end{array}$ \\
\hline $\begin{array}{l}\text { Preocupación } \\
\text { excesiva por } \\
\text { diferentes cosas }\end{array}$ & $66(22,4)[17,8-27,6]$ & $108(36,7)[31,2-42,5]$ & $74(25,2)[20,3-30,5]$ & $\begin{array}{c}46(15,6)[11,6- \\
20,3]\end{array}$ \\
\hline $\begin{array}{l}\text { Dificultad para } \\
\text { relajarse }\end{array}$ & $72(24,4)[19,6-29,8]$ & $108(36,7)[31,2-42,5]$ & $79(26,8)[21,8-32,3]$ & $\begin{array}{c}35(11,9)[8,43- \\
16,1]\end{array}$ \\
\hline $\begin{array}{l}\text { Intranquilidad, sin } \\
\text { quedarse quieto }\end{array}$ & $115(39,1)[33,5-44,9]$ & $98(33,3)[27,9-39,0]$ & $53(18,0)[13,8-22,9]$ & $\begin{array}{c}28(9,5)(6,42- \\
13,4]\end{array}$ \\
\hline $\begin{array}{l}\text { Irritado o enfadado } \\
\text { con facilidad }\end{array}$ & $74(25,1)[20,3-30,5]$ & $124(42,1)[36,4-48,0]$ & $69(23,4)[18,7-28,7]$ & $\begin{array}{c}27(9,1)[6,14- \\
13,0]\end{array}$ \\
\hline $\begin{array}{l}\text { Temor, como si fuera } \\
\text { a suceder algo terrible }\end{array}$ & $85(28,9)[23,8-34,4]$ & $89(30,2)[25,0-35,8]$ & $78(26,5) 2[1,5-31,9]$ & $\begin{array}{c}42(14,2)[10,4- \\
18,8]\end{array}$ \\
\hline
\end{tabular}

Tabla 3. Respuestas a la escala "Generalized Anxiety Disorder 7-item".

Tabla 4. Factores asociados a probables trastornos de ansiedad generalizada.

\begin{tabular}{|c|c|c|c|c|}
\hline \multicolumn{2}{|l|}{ Variable } & OR & [IC95\%] & $\mathbf{P}$ \\
\hline \multirow{2}{*}{ Genero } & Masculino & \multicolumn{3}{|c|}{1} \\
\hline & Femenino & 3,29 & {$[1,95-5,54]$} & $<0,001$ \\
\hline \multirow{5}{*}{ Rango etario } & $21-30$ & \multicolumn{3}{|c|}{1} \\
\hline & $31-40$ & 1,00 & {$[0,59-1,69]$} & 0,98 \\
\hline & $41-50$ & 0,89 & {$[0,38-2,10]$} & 0,80 \\
\hline & $51-60$ & 0,17 & {$[0,03-0,80]$} & 0,02 \\
\hline & $61-70$ & 0,23 & {$[0,02-2,03]$} & 0,19 \\
\hline \multicolumn{2}{|l|}{ Presentar humedad en las manos } & 7,77 & {$[4,38-13,7]$} & $<0,001$ \\
\hline \multicolumn{2}{|l|}{ Dificultad para dormir } & 6,99 & {$[3,82-12,80]$} & $<0,001$ \\
\hline \multicolumn{2}{|l|}{ Temblores musculares o tics nerviosos } & 6,28 & {$[3,21-12,2]$} & $<0,001$ \\
\hline \multicolumn{2}{|l|}{ Jaquecas, dolores de cabeza } & 6,00 & {$[3,41-10,56 \mid]$} & $<0,001$ \\
\hline \multicolumn{2}{|l|}{ Angustia al pensar que tiene que trabajar } & 5,98 & {$[3,00-11,9]$} & $<0,001$ \\
\hline \multicolumn{2}{|l|}{ Estrés desde que se inició la pandemia } & 5,96 & {$[3,15-13,98]$} & $<0,001$ \\
\hline \multicolumn{2}{|l|}{ Temor de llevar el virus a casa } & 5,88 & {$[3,42-10,10]$} & $<0,001$ \\
\hline \multicolumn{2}{|l|}{ Cansancio extremo o agotamiento } & 5,82 & {$[3,25-10,43]$} & $<0,001$ \\
\hline \multicolumn{2}{|l|}{ Nerviosismo con las noticias del COVID-19 } & 5,73 & {$[3,74-9,11]$} & $<0,001$ \\
\hline \multicolumn{2}{|l|}{ Pinchazos en el cuerpo } & 5,67 & {$[3,32-9,68]$} & $<0,001$ \\
\hline \multicolumn{2}{|l|}{ Incomodidad al pensar en el COVID-19 } & 5,49 & {$[2,68-11,26]$} & $<0,001$ \\
\hline \multicolumn{2}{|l|}{ Respiración entrecortada } & 5,36 & {$[3,10-9,26]$} & $<0,001$ \\
\hline \multicolumn{2}{|l|}{ Disminución del interés sexual } & 5,05 & {$[2,98-8,54]$} & $<0,001$ \\
\hline \multicolumn{2}{|l|}{ Indigestión, molestias gastrointestinales } & 5,03 & {$[3,01-8,40]$} & $<0,001$ \\
\hline \multicolumn{2}{|l|}{ Disminución del apetito } & 4,73 & {$[2,70-8,25]$} & $<0,001$ \\
\hline \multicolumn{2}{|l|}{ Renunciar al trabajo para proteger la familia } & 4,39 & {$[2,63-7,32]$} & $<0,001$ \\
\hline \multicolumn{2}{|l|}{ Pesadillas con el COVID-19 } & 3,98 & {$[2,39-6,64]$} & $<0,001$ \\
\hline \multicolumn{2}{|l|}{ No desear levantarse en la mañana para ir a trabajar } & 3,75 & {$[2,28-6,17]$} & $<0,001$ \\
\hline
\end{tabular}




\begin{tabular}{|c|c|c|c|}
\hline Temor familiar que regrese a casa infectado & 3,71 & {$[1,73-7,94]$} & $<0,001$ \\
\hline Tendencia a comer, beber o fumar más de lo habitual & 3,61 & {$[3,41-10,56]$} & $<0,001$ \\
\hline Temer la necesidad de acudir como paciente & 3,07 & {$[1,42-6,62]$} & $<0,001$ \\
\hline Temor al COVID-19 & 3,07 & {$[1,42-6,62]$} & $<0,001$ \\
\hline Temor de perder la vida con el COVID-19 & 3,03 & {$[1,29-6,19]$} & $<0,001$ \\
\hline Cambiar de casa para proteger la familia & 2,88 & {$[1,60-5,20]$} & $<0,001$ \\
\hline Intranquilidad desde que se inició la pandemia & 2,75 & {$[1,59-4,77]$} & $<0,001$ \\
\hline Decepcionado con el trabajo médico & 2,62 & {$[1,56-4,40]$} & $<0,001$ \\
\hline Percibir discriminación por su trabajo & 2,30 & {$[1,42-3,72]$} & $<0,001$ \\
\hline Haber creído tener síntomas del COVID-19 & 2,44 & {$[1,51-3,95]$} & $<0,001$ \\
\hline Sentirse protegido por sus empleadores & 0,47 & {$[0,24-0,93]$} & 0,030 \\
\hline Medidas gubernamentales suficientes & 0,43 & {$[0,23-0,82]$} & 0,010 \\
\hline Datos informados por las autoridades como confiables & 0,26 & {$[0,07-0,91]$} & 0,036 \\
\hline
\end{tabular}

\section{DISCUSIÓN}

Los trabajadores del área de la salud que laboran en medio de brotes epidémicos siempre van a estar expuestos a un mayor riesgo de adquirir la infección. Así se observó con COVID-19 e influenza H1N1, entre otras ${ }^{14,27}$. También van a tener mayor riesgo de presentar alteración en la salud mental. Un estudio realizado por $\mathrm{Lu}$ et $\left.a\right|^{28}$ en 2299 profesionales, 2042 médicos y 257 administrativos hospitalarios durante la pandemia COVID-19 señaló que los primeros tuvieron más presencia de temor, así como ansiedad y depresión cuando se midieron con la escala de ansiedad de Hamilton [HAMA] y la escala de depresión de Hamilton [HAMD]. A las condiciones laborales que usualmente incluyen numerosas horas continuas de actividad profesional, se suman pensamientos, percepciones, sentimientos y actuaciones que se convierten en estresores, que contribuyen a deteriorar el bienestar emocional, social y la productividad ${ }^{29-34}$.

La ansiedad es un estado fisiológico que responde a mecanismos neuroendocrinos y aflora cuando el organismo está bajo condiciones de riesgo o desventaja ${ }^{30,34}$. La ansiedad es una sensación displacentera, compuesta de manifestaciones somáticas o viscerales [involucrando diferentes órganos y sistemas], expresivas [posturales, faciales, vocales o respiratorias] y subjetivas [internas, personales, afectivas y vivenciales], que se pueden presentar combinadas o aisladas ${ }^{35}$. Cuando todas o algunas de esas manifestaciones se hacen crónicas o muy intensas, se llega a un cuadro patológico que puede interferir con el funcionamiento cotidiano del individuo y es denominado TAG, afectación mental que se cuenta entre las de mayor impacto sobre la salud en el siglo XXI, con asociación a disminución en la productividad laboral y académica ${ }^{18,28,31,32,36}$.

En el estudio observamos que el $38 \%$ de los participantes presentaron ansiedad, algo menor a lo observado en un estudio realizado por Lai et a ${ }^{16}$ también bajo la pandemia COVID-19 en la provincia de Hubei y ciudad de Wuhan, donde encontraron $44,6 \%$. El común denominador que eleva la presencia de ansiedad en los profesionales de la salud en momentos de epidemias es el temor ${ }^{15,37-39}$ En nuestro estudio, el $84 \%$ de los médicos generales informó temor al COVID-19. En trabajadores de la salud expuestos a la misma pandemia en China, encontraron elevada proporción de depresión, insomnio, angustia y ansiedad: el $70 \%$ informaron presentar temor ${ }^{16}$. En el estudio se observó que varios factores psicosociales, especialmente temor a enfermar por COVID-19 o de llevar la enfermedad a la familia, la percepción de discriminación, la sensación de angustia del trabajo diario y las noticias, se asociaron a ansiedad. Xiao et $a l^{14}$, encontraron que el personal médico chino que estaba tratando a pacientes con COVID-19 entre enero y febrero del 2020, tenía niveles de ansiedad, estrés y autoeficacia, que dependían de la calidad del sueño y del apoyo social.

La definición básica de temor o miedo es la sensación de angustia provocada por un peligro real o imaginario. Es una respuesta autónoma que no es 
activada de manera consciente ${ }^{40}$. Si bien es una experiencia individual, puede ser compartida como evento emocional de tipo social y circular a través de grupos. Al igual que muchas otras emociones, puede ser contagiosa y expandirse rápidamente, afectando el bienestar social ${ }^{15,19}$. La primera reacción de la humanidad frente a las epidemias es el temor al sufrimiento y a la muerte, con pérdida del bienestar emocional. Así se ha documentado en eventos de lepra, peste bubónica, $\mathrm{VIH} / \mathrm{sida}$, tuberculosis, viruela, cólera, SARS, ébola, entre otras ${ }^{12,13,39,41}$. La actual pandemia de COVID-19 no es la excepción ${ }^{42}$.

Lo encontrado en el estudio se explica por cambios fisiopatológicos. La integración de los componentes bioquímicos y hormonales del temor se controlan en el complejo amigdalino, constituido por núcleos neuronales ubicados en los lóbulos temporales. El más directamente implicado es el núcleo central, que envía axones al hipotálamo, mesencéfalo, protuberancia y bulbo. Cuando es activado, se provocan respuestas neurovegetativas y hormonales, con liberación de corticoesteroides y noradrenalina. El temor es una reacción en cadena que prepara al organismo automáticamente, con aumento del flujo sanguíneo, frecuencia cardíaca y un estado de excitación adrenérgico ${ }^{18,40,43}$.

En el estudio, más de la mitad de los participantes presentaban dificultades para conciliar el sueño, cefalea, molestias gastrointestinales, sensación de cansancio o reducción del interés sexual, mientras que entre el $20 \%$ y $30 \%$, tenían respiración entrecortada o ahogo, disminución del apetito, temblores musculares, tendencia a humedad en las manos. Estas son expresiones biológicas de estrés que también se explican desde la psiconeurobiología, que enfatiza la relación mente, pensamiento, emociones y reacciones corporales $^{14,35,40}$.

Los TAG pueden ser de corta duración o prolongada $^{18}$. En los profesionales de la salud pueden ser generados por la presión laboral, cognitiva o social, la sensación de frustración, la discriminación, la estigmatización o el aislamiento, las emociones negativas que subyacen en la relación médico/paciente, el estado físico-mental de agotamiento y el contacto que se tenga con sus familias $32-34,44$.

La evolución de la pandemia es elemento sustancial para la conservación del nivel de temor ${ }^{41}$. Los TAG pueden llevar a ataques de pánico que suelen demorar desde minutos hasta pocas horas ${ }^{17}$. En las epidemias los ataques de pánico suelen ser influenciados por noticias inadecuadamente presentadas, las cifras de morbilidad o mortalidad, la pérdida de familiares o amigos, la salida de control de la situación social o económica y por el colapso en la capacidad de atención por el sistema de salud $^{15,39}$.

En el momento de aplicar el formulario del presente estudio, en el Caribe colombiano se estaba en la fase inicial de confinamiento, con pocos casos diagnosticados, sin mortalidad entre los profesionales de la salud a nivel nacional, pero con una masiva y constante información noticiosa sobre lo desbordado de la entidad en otros países, especialmente China, Italia y España, y con la expectativa de comportamiento similar o peor ${ }^{42}$. Las epidemias no son estáticas, cambian día a día, se inician y se reducen súbitamente, induciendo diferentes reacciones psicobiológicas de los profesionales, sobre todo síntomas depresivos, insomnio, tristeza, negación e imposibilidad de utilizar herramientas cognitivas de afrontamiento, entre otros ${ }^{14}$. El temor y la ansiedad conllevan agotamiento y estrés, los cuales deterioran la calidad de atención, la habilidad para la toma de decisiones médicas, la capacidad de discernir entre los diagnósticos diferenciales, lo que induce a desenlaces negativos en el bienestar personal ${ }^{31}$.

En el estudio se encontró que el género femenino con respecto al masculino se asoció tres veces a mayor presencia de probable TAG. Un estudio en China, también en la pandemia COVID-19, indicó que el temor y la ansiedad estuvieron más presentes en mujeres que en hombres, enfermeras que en médicos; fueron factores asociados a su presencia: género femenino, ser estudiante y la pobre percepción de salud ${ }^{16}$.

Diversos autores han señalado que, en general, la ansiedad es informada por las mujeres dos o tres veces más frecuentemente que los varones ${ }^{18,29,45}$. No 
solo existen diferencias en las prevalencias, también en la expresión de los síntomas, el curso de la enfermedad, la respuesta a las intervenciones terapéuticas y la disposición para acudir a consulta psicológica o médica. Cada vez hay más pruebas de diferencias entre sexos con respecto a la anatomía cerebral, la síntesis de neurotransmisores, la arquitectura neuroquímica, el influjo hormonal, la respuesta a estresores sociales, los condicionantes culturales y a los estímulos ambientales ${ }^{45}$.

Además del factor ser mujer, se han señalado como asociados a ansiedad: los rasgos de la personalidad, las estrategias inadecuadas de afrontamiento, las alteraciones psicológicas previas, los aspectos cualitativos de la red social o familiar y la presencia de eventos estresantes; las pandemias, como el COVID-19, son fuertemente estresantes ${ }^{11}$.

Se encontró que sentirse protegido por los empleadores, considerar suficientes las medidas gubernamentales y confiar en las cifras oficiales se asociaron con menor presencia de probable TAG. Adib Ibrahim et $\mathrm{al}^{30}$, en profesionales de la salud, identificaron que estresores psicosociales como la influencia en el trabajo, la claridad de los roles, las recompensas y la satisfacción, explicaron más del $50 \%$ de la variación en la productividad de la atención médica.

Una de las características de las pandemias es lo desconocido. La ausencia de precisión científica temprana en cuanto a la propagación, contagiosidad, mortalidad e intervenciones, han sido el común denominador ${ }^{41}$. Lo desconocido acarrea temor y ansiedad, presentándose un círculo que potencializa la pérdida del bienestar individual, contándose como secuelas las enfermedades cardiovasculares, psiquiátricas, los trastornos del comportamiento alimentario y los disturbios del sueño ${ }^{32}$. Están disponibles guías dirigidas a la comunidad y profesionales que buscan reducir ansiedad, temor y prevenir el pánico ${ }^{46}$.

El estudio tiene como fortaleza ser de los primeros en aproximarse a valorar probable TAG en médicos generales del Caribe en momentos de pandemia, con un largo número de variables psicosociales y psicosomáticas. La forma virtual y masiva de invitación y participación puede ser fortaleza y debilidad al tiempo, así como permite llegar rápidamente a muchas áreas geográficas, impide validar el cumplimiento de los criterios de inclusión y la generación de sesgos. Posee las limitaciones propias de los estudios transversales, establece asociaciones estadísticas y no causalidades. Aunque se incluyó un número de participantes mayor al tamaño de la muestra, puede existir sobreestimación o subestimación en los resultados, ante la carencia de datos exactos de la población de médicos generales en el Caribe colombiano.

Se recomienda a los funcionarios sanitarios y gubernamentales realizar con prudencia los mensajes, instrucciones o medidas obligatorias en momentos de epidemia. El aislamiento preventivo se puede convertir en estresante, favoreciendo temor o ansiedad si se aplica excesivamente ${ }^{10}$. Las medidas sanitarias deben propender por reducir la ansiedad, por ello es fundamental considerar las diferencias en los comportamientos entre países, de acuerdo a exposición, geografía o climas, propagación y medidas preventivas ${ }^{42}$.

El Estado y las instituciones públicas o privadas de atención en salud deben oportunamente proporcionar los implementos necesarios de bioseguridad, así como ofertar educación para abordar todas las situaciones que rodean al evento pandémico; además, deben minimizar el temor, la ansiedad y evitar los brotes de pánico. El temor y la ansiedad hacen parte de la vida. Por eso, los médicos deben usar herramientas de afrontamiento y acudir a apoyo o valoración de su salud mental, de ser necesario ${ }^{15,27}$. Los casos probables de TAG, identificados con herramientas de cribado como el GAD-7, se deben valorar por psiquiatras, quienes, de acuerdo con las guías diagnósticas del CIE-10 o del DSM-V, llegarán al diagnóstico y adelantarán las intervenciones terapéuticas necesarias.

Se ameritan más estudios en médicos del Caribe colombiano en epidemias para precisar la influencia de aspectos psicobiológicos y psicosociales que deterioran el bienestar mental y favorecen la ansiedad, con fines de generar medidas preventivas y terapéuticas. Se concluye que en más de la mitad del grupo de médicos generales evaluados se 
encontraron sentimientos, pensamientos, percepciones, subjetividades y actuaciones negativas con respecto al COVID-19. Además, estrés, nerviosismo, temor, cansancio, incomodidad personal y familiar con respecto al COVID-19, decepción laboral y percepción de discriminación social, se asociaron con mayor presencia de probable TAG; mientras que sentirse protegidos por los empleadores, percibir como suficientes las medidas gubernamentales y considerar como creíble la información entregada por las autoridades se asociaron a menor presencia de probable TAG.

\section{AGRADECIMIENTOS}

Al grupo de médicos generales del Caribe colombiano y de todo el país que diligenciaron el formulario.

A los siguientes integrantes del Grupo de investigación Salud de la Mujer, que participaron incentivando la convocaría: Mejía-Mantilla A, Contreras-Saldarriaga J, Mercado-Lara M, QuintanaGuardo F, Redondo-Mendoza V, Dávila-Ruiz R, Monterrosa-Blanco A, Ahumada-Romero D, Romero-Martínez G, Romero-Martínez S, LópezGarcía T, Pinzón-Llanos J, Flores-Monterrosa Chabeli. A su vez, a Teresa Beltrán-Barrios que realizó ajustes gramaticales y de estilo.

\section{DECLARACIÓN SOBRE CONFLICTO DE INTERESES}

Los autores declaran que no existe conflicto de intereses

\section{CONTRIBUCIÓN DE LOS AUTORES}

Primer autor: diseño del estudio, análisis estadístico, escritura, revisión final y aprobación.

Segundo y tercer autor: trabajo de campo, análisis estadístico, escritura, revisión final y aprobación.

\section{REFERENCIAS BIBLIOGRÁFICAS}

1. Li X, Geng M, Peng Y, Meng L, Lu S. Molecular immune pathogenesis and diagnosis of COVID-19. J Pharm Anal. 2020 April;10(2):102-108. Doi: https://doi.org/10.1016/j.jpha.2020.03.001

2. Lake MA. What we know so far: COVID-19 current clinical knowledge and research. Clin Med (Lond). 2020 Mar;20(2):124-127. Doi: https://doi.org/10.7861/clinmed.2019-coron

3. PAHO/WHO. WHO characterizes COVID-19 as a pandemic [Internet]. Ginebra. [consultado 2020 abril 17]. Disponible en: https://www.paho.org/hq/index.php?option=com_ content\&view=article\&id=15756: who-

characterizes-covid-19-as-a-

pandemic\&ltemid=1926\&lang=en

4. World Health Organization. Coronavirus (CoV) [Internet.] Ginebra. [Consultado 2020 abril 20]. Disponible en: https://www.who.int/healthtopics/coronavirus/coronavirus\#tab=tab_1

5. World Health Organization. Emergencies preparedness, response. Novel Coronavirus-China. [Internet.] Ginebra. [consultado 2020 abril 19]. Disponible en: https://www.who.int/csr/don/12january-2020-novel-coronavirus-china/en/

6. World Health Organization. Strengthening health security by implementing the International Health Regulations. [Internet.] Ginebra. [Consultado 2020 abril 17]. Disponible en: https://www.who.int/news-room/q-a-detail/whatare-the-international-health-regulations-andemergency-committees

7. Accini-Mendoza JL, Beltrán N, Nieto-Estrada VH, Ramos-Bolaños E, Pizarro-Gómez C, Rebolledo CE, et al. Declaration of consensus in critical medicine for multidisciplinary care of the patient with a suspected or confirmed diagnosis of covid-19. Acta Colombiana de Cuidado Intensivo. 2020. Disponible online: 17 April $2020 . \quad$ Doi: https://doi.org/10.1016/j.acci.2020.04.003

8. Centers for Disease Control and Prevention. CDC. Coronavirus disease 2019 (COVID-19). Stress and coping. [Internet]. [Consultado 2020 abril 17]. 
Disponible

en:

https://www.cdc.gov/coronavirus/2019-ncov/dailylife-coping/managing-stress-anxiety.html

9. Centers for Disease Control and Prevention. CDC. Coronavirus disease 2019 (COVID-19). How to protect yourself \& others. [Internet]. [Consultado 2020 abril 17]. Disponible en: https://www.cdc.gov/coronavirus/2019ncov/prevent-getting-sick/prevention.html

10. Brooks SK, Webster RK, Smith LE, Woodland L, Wessely S, Greenberg N, et al. The psychological impact of quarantine and how to reduce it: rapid review of the evidence. Lancet. 2020 Mar 14;395(10227):912-920. Doi: https://doi.org/10.1016/S0140-6736(20)30460-8

11. Mohindra RRR, Suri V, Bhalla A, Singh SM. Issues relevant to mental health promotion in frontline health care providers managing quarantined/isolated COVID19 patients. Asian J Psychiatr. 2020 Jun;51:102084. Doi: https://doi.org/10.1016/j.ajp.2020.102084

12. Khalid I, Khalid TJ, Qabajah MR, Barnard AG, Qushmaq I. Healthcare workers emotions, perceived stressors and coping strategies during a MERS-CoV outbreak. Clin Med Res. 2016 Mar;14(1):7-14.

Doi: https://doi.org/10.3121/cmr.2016.1303

13. Verma S, Mythily S, Chan YH, Deslypere JP, Teo EK, Chong S. Post-SARS psychological morbidity and stigma among general practitioners and traditional Chinese medicine practitioners in Singapore. Ann Acad Med Singapore. 2004 Nov;33(6):743-748. https://pubmed.ncbi.nlm.nih.gov/15608831/

14. Xiao H, Zhang Y, Kong D, Li S, Yang N. The effects of social support on sleep quality of medical staff treating patients with coronavirus disease 2019 (COVID-19) in january and february 2020 in China. Med Sci Monit. 2020 Mar 5;26:e923549. Doi: https://doi.org/10.12659/MSM.923549

15. Shultz JM, Althouse BM, Baingana F, Cooper JL, Espinola M, Greene MC, et al. Fear factor: The unseen perils of the Ebola outbreak. Bull At Sci.
2016;72(5):304-310.

Doi: https://doi.org/10.1080/00963402.2016.1216515

16. Lai J, Ma S, Wang Y, Cai Z, Hu J, Wei N, et al. Factors Associated With Mental Health Outcomes Among Health Care Workers Exposed to Coronavirus Disease 2019. JAMA Netw Open. 2020 Mar 2;3(3):e203976. Doi: https://doi.org/10.1001/jamanetworkopen.2020.3 976

17. Thibaut F. Anxiety disorders: a review of current literature. Dialogues Clin Neurosci. 2017 Jun;19(2):87-88.

https://pubmed.ncbi.nlm.nih.gov/28867933/

18. Academia Nacional de Medicina de México [ANMM]. El trastorno de ansiedad generalizada. Clínica Terapéutica de la Academia Nacional de Medicina. 2012;21(3):1-2. http://www.scielo.org.mx/pdf/facmed/v56n4/v56n 4a9.pdf

19. Dunsmoor JE, Paz R. Fear generalization and anxiety: behavioral and neural mechanisms. Biol Psychiatry. 2015 Sep 1;78(5):336-343. Doi: https://doi.org/10.1016/j.biopsych.2015.04.010

20. Dymond S, Dunsmoor JE, Vervliet B, Roche B, Hermans D. Fear generalization in humans: systematic review and implications for anxiety disorder research. Behav Ther. 2015 Sep;46(5):56182. Doi: https://doi.org/10.1016/j.beth.2014.10.001

21. Beard C, Björgvinsson T. Beyond generalized anxiety disorder: psychometric properties of the GAD-7 in a heterogeneous psychiatric sample. J Anxiety Disord. 2014 Aug;28(6):547-52. Doi: https://doi.org/10.1016/j.janxdis.2014.06.002

22. García-Campayo J, Zamorano E, Ruiz MA, Pardo A, Pérez-Páramo $M$, López-Gómez $V$, et al. Cultural adaptation into Spanish of the generalized anxiety disorder-7 (GAD-7) scale as a screening tool. Health Qual Life Outcomes. 2010;8:8. Doi: https://doi.org/10.1186/1477-7525-8-8

23. El Espectador.com En Colombia hay un médico por cada 846 habitantes. Prensa (Colombia) 27 Febrero-2012. [Internet]. [Consultado 2020 abril 
17].

Disponible

en: https://www.elespectador.com/noticias/salud/colo mbia-hay-un-medico-cada-846-habitantes-articulo329003

24. López L. El país necesita más personal en salud. Prensa (Colombia) 24 mayo-2018. [Internet]. [Consultado 2020 abril 17]. Disponible en: https://www.elcolombiano.com/colombia/salud/el -pais-necesita-mas-personal-en-salud-NC8747438

25. Sánchez AM. Por cada 1.000 habitantes en Colombia, hay alrededor de 1,5 médicos generales. Prensa (Colombia) 25 de marzo de 2020. [Internet]. [Consultado 2020 abril 17]. Disponible en: https://www.larepublica.co/economia/por-cada1000-habitantes-en-colombia-hayaproximadamente-15-medicos-generales-2982596

26. República de Colombia. Ministerio de Salud. Resolución 8430 de 1993. [Internet]. [Consultado 2020 abril 17]. Disponible en: https://www.minsalud.gov.co/sites/rid/Lists/Bibliot ecaDigital/RIDE/DE/DIJ/RESOLUCION-8430-DE1993.PDF

27. Pryluka D, Lopardo G, Daciuk L, Stecher D, Bonvehi $P$. Severe acute respiratory disease in health-care workers during the influenza H1N1 pandemic in Argentina. J Infect Dev Ctries. 2013 Jan 15;7(1):36-40.

Doi: https://doi.org/10.3855/jidc.2368

28. Lu W, Wang H, Lin Y, Li L. Psychological status of medical workforce during the COVID-19 pandemic: A cross-sectional study. Psychiatry Res. 2020 Apr 4;288:112936.

Doi: https://doi.org/10.1016/j.psychres.2020.112936

29. Chen Q, Zhang Y, Zhuang D, Mao X, Mi G, Wang $D$, et al. Health anxiety in medical employees: A multicentre study. J Int Med Res. 2019 Oct;47(10):4854-4861. Doi: https://doi.org/10.1177 / 0300060519872310

30. Adib Ibrahim M, Abdul Aziz A, Suhaili NA, Zahid Daud A, Naing L, Abdul Rahman H. A Study into psychosocial work stressors and health care productivity. Int J Occup Environ Med. 2019
Oct;10(4):185-193.

Doi: https://doi.org/10.15171/ijoem.2019.1610

31. Dewa CS, Loong D, Bonato S, Thanh NX, Jacobs P. How does burnout affect physician productivity? A systematic literature review. BMC Health Serv Res. 2014 Jul 28;14(325):1-10. Doi: https://doi.org/10.1186/1472-6963-14-325

32. Erdur B, Ergin A, Turkcuer I, Parlak I, Ergin N, Boz B. A study of depression and anxiety among doctors working in emergency units in Denizli, Turkey. Emerg Med J. 2006 Oct;23(10):759-63. Doi: https://doi.org/10.1136/emj.2006.035071

33. Daniels K, Swartz L. Understanding health care workers' anxieties in a diversifying world. PLoS Med. $2007 \quad$ Nov 13;4(11):e319. Doi: https://doi.org/10.1371/journal.pmed.0040319

34. Kwiatosz-Muc M, Fijałkowska-Nestorowicz A, Fijałkowska M, Aftyka A, Kowalczyk M. Stress prevalence and stressors among anesthesiology and intensive care unit workers: A multicentre survey study. Aust Crit Care. 2018 Nov;31(6):391-395. Doi: https://doi.org/10.1016/j.aucc.2017.11.001

35. Bonet J. A short review of four key physiopathological mechanisms for psychosomatic medicine. [Article in Spanish]. Vertex. 2019 MayJun;(145):185-

194.https://www.researchgate.net/publication/33 9080857_A_short_review_of_four_key_physiopath ological_mechanisms_for_psychosomatic_medicin e

36. Matsuishi K, Kawazoe A, Imai H, Ito A, Mouri K, Kitamura $\mathrm{N}$, et al. Psychological impact of the pandemic (H1N1) 2009 on general hospital workers in Kobe. Psychiatry Clin Neurosci. 2012 Jun;66(4):353-360.

Doi: https://doi.org/10.1111/j.1440-1819.2012.02336.x

37. Maunder R, Hunter J, Vincent L, Bennett J, Peladeau $N$, Leszcz $M$, et al. The immediate psychological and occupational impact of the 2003 SARS outbreak in a teaching hospital. CMAJ. 2003 May 
https://www.ncbi.nlm.nih.gov/pmc/articles/PMC15 4178/

38. Chua SE, Cheung V, Cheung C, McAlonan GM, Wong JW, Cheung EP, et al. Psychological effects of the SARS outbreak in Hong Kong on high-risk health care workers. Can J Psychiatry. 2004 Jun;49(6):391393.

Doi:

https://doi.org/10.1177/070674370404900609

39. Lermann W. The Man and his epidemics through the History. Rev Chile Infectol. 2003;20(Supp 2):1317. Doi: http://dx.doi.org/10.4067/S071610182003020200003

40. Garcia R. Neurobiology of fear and specific phobias. Learn Mem. 2017 Aug 16;24(9):462-471. Doi: https://doi.org/10.1101/Im.044115.116

41. Galeano D. Médicos y policías durante la epidemia de fiebre amarilla (Buenos Aires, 1871). Salud colectiva. 2009;5:107-120. https://www.scielosp.org/article/scol/2009.v5n1/1 07-120/

42. Amariles P1, Granados J2, Ceballos M2, Montoya CJ. COVID-19 in Colombia endpoints. Are we different, like Europe? Res Social Adm Pharm. 2020 Mar 31. pii: S1551-7411(20)30287-4. Doi: https://doi.org/10.1016/j.sapharm.2020.03.013

43. Kozlowska K, Walker P, McLean L, Carrive P. Fear and the defense cascade: clinical implications and management. Harv Rev Psychiatry. 2015 JulAug;23(4):263-87. Doi: https://doi.org/10.1097/HRP.0000000000000065

44. Kang L, Li Y, Hu S, Chen M, Yang C, Yang BX, et al. The mental health of medical workers in Wuhan, China dealing with the 2019 novel coronavirus. Lancet Psychiatry. 2020 Mar;7(3):e14. Doi: https://doi.org/10.1016/S2215-0366(20)30047-X

45. Arenas MC, Puigcerver A. Diferencias entre hombres y mujeres en los trastornos de ansiedad: una aproximación psicobiológica. Escritos de Psicología. 2009;3(1):2029.http://scielo.isciii.es/scielo.php?script=sci_artte xt\&pid=S1989-38092009000300003
46. Helpguideorg international [HelpGuide]. Coronavirus anxiety: coping with stress, fear, and worry. [Internet.] Estados Unidos. [Consultado 2020 abril 19]. Disponible en: https://www.helpguide.org/articles/anxiety/corona virus-anxiety.htm 